\title{
Risk Assessment tool for Religious Mass Gathering Events of India
}

\section{Upasana Sharma, Sankara Sarma}

\author{
SCTIMST, India
}

\section{Objective}

To develop a risk assessment tool to assess the public health and environmental risks associated with religious mass gathering events of Tamil Nadu, a state in the southern part of India

\section{Introduction}

In spite of the fact that mass gatherings are an undeniably regular element of our society attended by huge crowds yet such occasions are not very well understood. Even though such gatherings are accumulations of "well people", vast number of people associated with mass gatherings can put a serious strain on the entire health care system [1].The public health implications of mass gathering events include a potential increased risk for disease transmission because of the variability and mobility of those attending the event and increased media attention. Risk assessment for mass gathering events is crucial to identify the potential health hazards which aids in planning and response activities specific to the event [2]. Preparing for mass gatherings offer an opportunity to improve health service delivery, enhance health promotion and strengthen public health systems [3].

In India, many of the religious festivals are observed with mass gatherings and prayers. Large crowd participate in such festivals as participants to observe the unique rituals and also as spectators. Literature indicates that in India, we might be well equipped for response activities but the scientific concept of risk assessment i.e., to understand the existing risks, identify the risks, characterize the risks and plan for risk reduction strategies accordingly are at an infant stage .The little that has been done in the field of mass gatherings has generally focused on description of preparedness activities of single event, crowd control, prevention of stampedes with little attention to public health preparedness. The present project is an attempt to systemize the process of risk assessment by developing a risk assessment tool consisting of characteristics peculiar to planned religious mass gatherings of Indian context.

\section{Methods}

Qualitative approach was followed to identify the risks associated with mass gathering events and to identify the domains and items to be included in the risk assessment tool. Firstly, an extensive review of literature about the risks associated with the mass gathering events was done. Secondly, Key Informants $(n=20)$ involved in planning and management of religious mass gathering events in the State of Tamil Nadu, India were purposively identified and interviewed using a semi structured interview guide. Principle of redundancy was followed. Content/Thematic analysis was done using Atlas.ti software. Currently, the project is in the phase of obtaining content validity of the developed tool. Followed by this, a mobile application based upon the validated tool will be developed which will be further field tested for feasibility in a selected mass gathering event in Tamil Nadu. Using a self administered content validity questionnaire, the experts will be asked to assess the relevance of the items of the tool. Agreement proportions between the experts will be calculated. S-CVI (Scale Content Validity Index), index for inter-rater agreement (agreement proportion) and Kappa agreement coefficient will be calculated.

\section{Results}

A sum total of 48 unique health risks have been identified. Stampedes, fire accidents, structural collapse, drowning, outbreak of communicable diseases, exacerbation of existing medical illnesses (like cardiac diseases, asthma etc) etc are the some of the health risks identified. Six domains (characteristics related to event, participant, environment, disaster preparedness, medical service preparedness and pre event planning activities) and 21 items have been generated from the content analysis of key informant interviews and literature review. 
ISDS 2019 Conference Abstracts

\section{Conclusions}

Some special events and unforeseen events occur in places of mass gatherings besides fixed places of worshipping .Such events cause more damage to human beings and property. Special events like idol procession, chariot pulling, fire walking, animal sacrificing happen pulling larger crowds within the mass gatherings. In order to inform all planning and delivery activities it is essential to understand the mass gathering context and risk assessments. This tool can be used by public health managers to identify key public health and environmental risks at the planning stage before the event takes off. At the planning stage, u se of this tool will help in putting the required measures in place in order to address the potential risks identified. The tool can be used as a guiding instrument during and after the event as well. The investigators further plan to develop a mobile based app from this risk assessment tool and test it out in a selected mass gathering event of the state of Tamil Nadu located in southern part of India. Feedback from public health managers about the mobile based risk assessment tool can be instrumental in further modifying the tool. By contributing to public health preparedness activities during mass gathering events in a country with poor resources like India, this research activity is an initiative that is expected to lead to health systems strengthening.

\section{References}

1. Arbon P. 2004. The development of conceptual models for mass-gathering health. Prehosp Disaster Med. 19(3), 208-12. PubMed https://doi.org/10.1017/S1049023X00001795

2. World Health Organization. Public health for mass gatherings: Key considerations. Geneva: WHO; 2015.

3. Tam JS, Barbeschi M, Shapovalova N, Briand S, Memish ZA, et al. 2012. Research agenda for mass gatherings: a call to action. Lancet Infect Dis. 12(3), 231-39. PubMed https://doi.org/10.1016/S14733099(11)70353-X

ISDS Annual Conference Proceedings 2019. This is an Open Access article distributed under the terms of the Creative Commons AttributionNoncommercial 4.0 Unported License (http://creativecommons.org/licenses/by-nc/3.0/), permitting all non-commercial use, distribution, and reproduction in any medium, provided the original work is properly cited. 